\title{
Canguilhem, Foucault y la ontología política del vitalismo 1
}

\author{
Canguilhem, Foucault and the \\ political ontology of vitalism
}

\author{
Francisco VÁzQUEZ GARcía \\ (Universidad de Cádiz)
}

Recibido: 01/04/2014

Aceptado: 12/11/2014

\section{Resumen}

En este artículo se defiende la tesis de que la noción de vida, en la obra de Foucault, no es sólo el correlato de prácticas históricas de poder y saber. Foucault sostiene una ontología vitalista, intermitente y no del todo explícita. La fuente de esta opción filosófica en Foucault no es nietzscheana ni deleuziana, sino que coincide en muchos aspectos con el vitalismo no esencialista de Georges Canguilhem. En primer lugar comparamos la relación de Canguilhem y de Foucault con la tradición kantiana, en su vertiente crítica y trascendental. En segundo lugar, comparamos la noción de vida en las propuestas filosóficas de Canguilhem y Foucault. Por último analizamos la procedencia canguilhemiana del concepto foucaultiano de normalización. Esta filiación se advierte también en el concepto de biopolítica, referido a un poder que actúa sobre la vida, imitando su funcionamiento. Este arraigo del pensamiento de Foucault en el vitalismo de su maestro permite resolver dos tensiones internas en la trayectoria del primero: la justificación de los movimientos de revuelta y el carácter autoformativo de la subjetividad.

Palabras clave: vida, vitalismo, ontología, Foucault, Canguilhem, biopolítica, subjetividad.

\footnotetext{
${ }^{1}$ Este trabajo se ha realizado gracias a la financiación de la Dirección General de Investigación del Ministerio de Ciencia e Innovación, dentro del proyecto "Vigilancia de fronteras, colaboración crítica y reconversión: un estudio comparado de la relación de la filosofía con las ciencias sociales en España y Francia (1940-1990)", referencia FFI2010-15196 (subprograma FISO)
} 


\begin{abstract}
In this paper we defend the thesis that the notion of life, in Foucault's work, is not only the historical correlate of power and knowledge practices. Foucault argues a Vitalist ontological position, however intermittent and not at all explicit. The source of this philosophical choice in Foucault is not Nietzsche or Deleuze, but it coincides in many respects with the non-essentialist Vitalism of Georges Canguilhem. First, we compare Canguilhem and Foucault's relation to the Kantian tradition, in its critical and transcendental aspects. Secondly, we confront the notion of life on the philosophical proposals of Canguilhem and Foucault. Finally we analyze the canguilhemian origin of the normalization foucauldian concept. This affiliation also regards the concept of biopolitics, referred to a power that acts on life, imitating its operation. This rooting of the thought of Foucault in the Vitalism of his master resolves two internal tensions in the path of the first: the justification of movements of revolt and self-formative character of subjectivity.
\end{abstract}

Keywords: life, Vitalism, ontology, Foucault, Canguilhem, biopolitics, subjectivity.

\title{
1. El descubrimiento del "Canguilhem perdido" y sus consecuencias para el estudio de las relaciones intelectuales entre Foucault y su maestro
}

Las relaciones intelectuales entre Foucault y Canguilhem vienen siendo exploradas desde hace mucho tiempo. Entre ambos pensadores, y esto fue reconocido por $\operatorname{ambos}^{2}$, tuvo lugar un fructífero intercambio recíproco de conceptos, motivos, actitudes y estilos interpretativos 3 . Sin afán de exhaustividad, pueden recordarse algunos de los hitos que enmarcan este movimiento de mutua importación y préstamo. Las críticas de Canguilhem a la psicología en su célebre conferencia de 1956, el análisis de lo normal y lo patológico en su tesis doctoral (1943) (con la primacía de lo patológico sobre lo normal y del error sobre la verdad), su historiografía no teleológica o sus estudios sobre el darwinismo, incidieron decisivamente en las obras de la primera etapa foucaultiana (los escritos sobre la psicología de la etapa prearqueológica, la Historia de la locura, El nacimiento de la clínica, Las palabras y las

\footnotetext{
2 Canguilhem, G.: "Sur l'Histoire de la folie en tant qu'événement”, Le Débat, 41 (1986), pp. 37-40 y Foucault, m.: "La vie: l'experience et la science", Dits et Écrits (en adelante D. E.), Paris, Gallimard, pp. 763-776.

3 Davidson, A.: "Sobre epistemología y arqueología: de Canguilhem a Foucault", en La aparición de la sexualidad. La epistemología histórica y la formación de los conceptos, Barcelona, Alpha Decay, 2004, p. 287; I. Cutro, A.: Michel Foucault. Tecnica e vita. Biopolitica e filosofía del bios, Nápoles, Bibliopolis, 2004, p. 18; Paltrinieri, L.: L'experience du concept. Michel Foucault entre épistémologie et histoire, Paris, Publications de la Sorbonne, 2012, p. 54.
} 
cosas). La metodología arqueológica de Foucault también estuvo en deuda con la historia de los conceptos armada por Canguilhem, en particular la distinción entre proposiciones verdaderas (decir la verdad) y régimen de verdad (estar en la verdad), instancia muy presente en $\mathrm{El}$ orden del discurso ${ }^{4}$. Al mismo tiempo, la segunda parte de Lo normal y lo patológico (sobre todo el apartado acerca de lo vital a lo social), recoge todo lo que dice Foucault sobre las condiciones institucionales de la medicina clínica (en El nacimiento de la clínica). Finalmente, puede recordarse que el concepto canguilhemiano de "ideología científica", desarrollado en la década de los setenta, se inspira tanto en Foucault como en Althusser.

Pero mi intención no es volver sobre este asunto; tampoco se trata de proponer un estudio sociofilosófico, como los que he realizado en otras ocasiones ${ }^{5}$. La aportación que presento, de orden puramente conceptual, se sitúa en continuidad con una nueva manera de enfocar las relaciones entre ambos autores, coincidiendo con una profunda renovación, iniciada a comienzos de este siglo, en la manera de interpretar a Canguilhem. Hasta entonces este autor era considerado como epistemólogo e historiador de las ciencias, seguidor de la metodología bachelardiana y precursor de Foucault. Esta lectura, auspiciada en la década de los sesenta por los discípulos de Althusser y de Lacan nucleados en torno al Cercle d'Épistémologie de l'ENS y a la revista Cahiers por l'Analyse, ha sellado durante más de treinta años el modo de acercarse a la obra de Canguilhem. Pues bien, tras el redescubrimiento entre la segunda mitad de los 90 y la primera década del siglo XXI, de un corpus de más de cien obras prácticamente desconocidas y editadas entre 1926 y 1939, incluyendo decenas de artículos y algún que otro libro 6 , la imagen de Canguilhem ha salido completamente transformada. Desde esas fechas el crecimiento de la literatura sobre el maestro de Foucault, especialmente en Francia, pero también en el mundo cultural angloamericano y en Alemania, ha sido espectacular; reuniones

\footnotetext{
4 Sobre este asunto existe toda una controversia. Véanse las posiciones contrapuestas de Balibar, E.: "Science et verité dans la philosophie de Georges Canguilhem", en AAVV.: Georges Canguilhem. Philosophe et historien des sciences, Paris, Albin Michel, 1993, pp. 58-76; Delaporte, F.: "Foucault, Canguilhem et les monstres", en Braunstein, J. F. (coord.): Canguilhem. Histoire des sciences et politique du vivant, Paris, PUF, 2007, pp. 91-112 y Delaporte, F.: "Histoire d'être 'dans le vrai'", en en Ferté, L., Jacquard, A. et Vermeren, P. (dir.): La formation de Georges Canguilhem. Un entre-deuxguerres philosophique, París, Hermann, 2013, pp. 341-354.

5 Vázquez García, F.: La filosofía española. Herederos y pretendientes. Una lectura sociológica (1963-1990), Madrid, Abada Editores, 2009 y Vázquez García, F.: Hijos de Dionisos. Sociogénesis de una vanguardia nietzscheana (1968-1985), Madrid, Biblioteca Nueva, 2014.

$6 \mathrm{El}$ inventario de este corpus fue realizado por Limoges, C.: "Critical Bibliography", en Delaporte, F. (ed.): A vital rationalist. Selected writings from Georges Canguilhem, New York, Zone Books, 1994, pp. 385-454. El primer trabajo que ponderó sus consecuencias fue el de Braunstein, J. F. : "Canguilhem avant Canguilhem", Revue d'histoire des sciences, 53, 2000, 1, pp. 9-26. Los textos están recogidos en el primer tomo de sus obras completas, en curso de edición: Canguilhem, G.: Oeuvres Complètes. Écrits philosophiques et politiques 1926-1939, Paris, Vrin, 2011.
} 
científicas y Congresos, monográficos de distintas revistas, libros individuales y colectivos, se han concertado para fabricar un nuevo Canguilhem. Se ha puesto de manifiesto que su interés por la epistemología y la historia de las ciencias fue en realidad tardío en su trayectoria, subordinado a una filosofía de la vida donde los aspectos prácticos, morales y políticos, primaban sobre las preocupaciones de la filosofía teórica7.

Esta deriva ha llevado a plantear de un nuevo modo las relaciones entre Foucault y Canguilhem. Entre las décadas de los 70 y los 90 esas conexiones se indagaban principalmente en el plano del método histórico y de la diferencia entre análisis arqueológico y análisis epistemológico. Aunque el examen de estos nexos no ha desaparecido de la literatura secundaria, el foco prioritario lo constituyen ahora los vínculos ontológicos y ético-políticos entre ambos proyectos filosóficos. Destacan en particular tres ámbitos de interrogación: las afinidades y divergencias entre ambos autores en relación con los conceptos de norma vital y social8; la implicación de ambos pensadores en la génesis del concepto de "biopolítica"9 $\mathrm{y}$, finalmente, la importancia de la noción de vida en ambas trayectorias ${ }^{10}$.

Mi punto de partida lo constituye un problema planteado por Béatrice Han en L'ontologie manquée de Michel Foucault ${ }^{11}$. Esta autora levanta acta de la tensión irresuelta, en la obra de Foucault, entre lo histórico y lo trascendental. La historización radical de todo fundamento trascendental, sea subjetivo (como el sujeto trascendental kantiano) u objetivo (como la Vida, el Trabajo o el Lenguaje), emprendida por el método arqueogenealógico, le condujo en sus dos primeras etapas a presentar al sujeto como un efecto de regímenes de verdad y dispositivos de poder anónimos e históricamente mudables. Sin embargo, el último Foucault parece recuperar una subjetividad autoformativa. Según Han, esta tensión, que lleva a bifurcar la

\footnotetext{
7 Limoges, C.: “L'épistémologie historique dans l'itineraire intellectual de Georges Canguilhem”, en AAVV.: Epistemology and history from Bachelard and Canguilhem to today's history of science, Berlín, Max Planck Institute for the History of Science, 2012, pp. 53-66.

8 Macherey, P.: (2009): De Canguilhem á Foucault, la force des norms, Paris, La Fabrique Éditions

9 Mühle, M.: Eine Genealogie der Biopolitik: Zum Begriff des Lebens bei Foucault und Canguilhem, Stuttgart, Merz Akademie, 2008; Mühle, M.: "Sobre la vitalidad del poder: una genealogía de la biopolítica a partir de Foucault y Canguilhem", en Lemm, V. (ed.): Michel Foucault: neoliberalismo y biopolitica, Santiago de Chile, Ediciones Universidad Diego Portales, 2010, pp. 397-432; Mühle, M.: "Histoire(s) de la vie de Canguilhem á Foucault", en AAVV.: Epistemology and history, op. cit, pp. 187-195 y Velasco Arias, G.: Genealogías de lo biopolitico. Normalidad y patología en el "momento foucaultiano", Madrid, Universidad Autónoma de Madrid, Tesis Doctoral (inédita), 2013

10 Le Blanc, G.: La vie humaine. Anthropologie et biologie chez Georges Canguilhem, Paris, PUF, 2002, pp. 199-217; Cutro, A., op. cit.; Paltrinieri, L., op. cit. y López, C.: "Georges Canguilhem: une autre manière d'approcher la notion de vie", en Ferté, L., Jacquard, A. et Vermeren, P. (dir.), op. cit., pp. 253-267.

11 Han, B.: L'ontologie manqué de Michel Foucault, Grenoble, Éditions Jérôme Millon, 1998, pp. 728 y $305-321$.
} 
lectura de Foucault en una clave antihumanista (borrado del sujeto en las configuraciones praxeológicas) o voluntarista (capacidad autogestante del sujeto) sólo puede resolverse de dos maneras. O quedándose en la perspectiva arqueogenealógica inicial -que Han califica de nietzscheana- renunciando al sujeto autopoiético, o reinterpretando su proyecto como ontología hermenéutica, en la estela heideggeriana. En este caso el pensamiento dejaría de identificarse con la actividad de un sujeto, mostrándose en cambio como una instancia antepredicativa y anónima, inherente a toda práctica. Al no proseguir decididamente ninguna de estas dos vías, Foucault quedaría, según Han, presa de los dobles antropológicos característicos de la episteme moderna y explorados por él mismo en Les mots et les choses.

Por mi parte, entiendo que es posible ir más allá de esa disyuntiva. Defiendo la tesis de que la "ontología esquivada" por Foucault -usando los términos de Han, latente en su obra y que no deja de asomar en algunos pasajes de la misma, es una ontología vitalista. Esto ya había sido defendido por Gilles Deleuze12; lo que este no había precisado es que ese vitalismo no es el de la voluntad de poder nietzscheana ni el del propio Deleuze, sino el elaborado por Georges Canguilhem. Sin embargo, el vitalismo racionalista sostenido por el maestro de Foucault aparece como una ontología paradójica, pues como se comprobará, Canguilhem niega la existencia de una verdad filosófica o de una apertura originaria a la verdad; considera que la filosofía no tiene que ver con la verdad sino con el valor. Su cometido no es descubrir sino evaluar. Sostiene pues el primado de la filosofía práctica.

La defensa del vitalismo racionalista de Canguilhem como impensado ontológico de la obra de Foucault, no sólo permite, según creemos, rebasar la tensión interna descrita por Han, conectando la subjetividad autoformativa del último Foucault con el viviente autogenerado evocado por Canguilhem. Al mismo tiempo posibilita utilizar a Foucault alejándolo de las lecturas realizadas en clave de "construccionismo social extremo". Esta vía, muy explotada desde los Cultural Studies, los Queer Studies, o las versiones del "programa fuerte" en sociología de la ciencia, emparentan a Foucault con un relativismo epistémico del que estaba, a nuestro parecer, muy alejado. En cambio, el vitalismo racionalista de Canguilhem, en su manera de entender la relación entre normas sociales y vitales, conduce al rechazo, tanto del construccionismo social extremo como de las orientaciones neonaturalistas en ciencias sociales, vinculadas al auge de la sociobiología o de las neurociencias ${ }^{13}$. Se entiende así, y el recurso a Foucault y a Canguilhem resulta aquí sumamente valioso, que los movimientos sociales y las iniciativas políticas ligadas a la vida y a la

12 Deleuze, G.: Foucault, Barcelona, Paidós, 1987, p. 122.

13 Sobre el rechazo de Canguilhem al construccionismo social al estilo de Bruno Latour, véase Bouveresse, J.: "Préface aux Oeuvres Complètes de Georges Canguilhem", en Canguilhem, G.: Oeuvres Complètes, op. cit., pp. 44-46. Sobre las reservas ante el afán expansivo de las neurociencias, Canguilhem, G.: "Le cerveau et la pensé", en AAVV.: Georges Canguilhem. Philosophe et historien des sciences, op. cit., pp. 11-33. 
identidad (como el movimiento LGTB o el de Diversidad Funcional), no están condenados a legitimarse filosóficamente a partir de la pobre alternativa entre el construccionismo relativista y el naturalismo biologicista.

\section{La herencia kantiana del maestro y del discípulo}

La filiación kantiana de Canguilhem ha sido glosada por un gran número de comentaristas 14 ; en el caso de Foucault fue él mismo, en el autoanálisis que publicó como voz de un diccionario bajo el seudónimo de Maurice Florence, quien subrayó ese vínculo ${ }^{15}$. Puede decirse que Foucault tomó de Canguilhem una manera de afrontar la actividad filosófica, consistente en desplazar la problemática kantiana de la unidad de la experiencia, de las condiciones a priori de la experiencia, llevándolas del plano trascendental al histórico.

Muy recientemente se ha descrito el particular recorrido kantiano surcado por Canguilhem desde su juventud ${ }^{16}$. Este se formó en una particular versión del kantismo; la ofrecida, entre finales del siglo XIX y primeras décadas del XX, por lo que se conoce como escuela del "análisis reflexivo". Este estilo de pensamiento, que aglutinó a filósofos hoy poco recordados, como Lagneau, Boutroux o Alain, tenía su punto de partida en el problema de la unidad de la experiencia. ¿Qué instancia trascendental es la que pone orden y unifica la dispersión originaria de lo fenoménico? Para lidiar con este asunto, los representantes del análisis reflexivo bosquejaron una lectura de la Crítica de la razón pura que combinaba al mismo tiempo lo epistémico y lo moral. Por eso se subrayaba la condición activa de las facultades implicadas en el uso teórico de la razón. Tanto la percepción como el juicio se afrontan en términos de acción. Son actividades por las que el espíritu se opone incesantemente a la materia, trasciende el plano de los hechos y los somete al imperio del valor. Se trata pues de una lectura que inserta un giro moral en el núcleo mismo de la analítica trascendental, fusionando el contenido de las dos primeras críticas, asimilando el entendimiento y la actividad judicativa a la acción de la voluntad y a la afirmación de la dignidad humana, por encima de lo dado, de los meros hechos.

\footnotetext{
14 Vermeren, P.: "Préface. Canguilhem, philosophe du parti pris", en Ferté, L., Jacquard, A. et Vermeren, P. (dir), op. cit., p. 19; Cornu, L.: "Le parti pris humain", en id., p. 110; David-Ménard, M.: "La limitation des champs de savoir chez Kant, Canguilhem et Foucault", en id., pp. 355-370, y especialmente, Roth, X.: Georges Canguilhem et l'unité de l'experience. Juger et agir 1926-1939, París, Vrin, 2013.

15 Foucault, M.: "Foucault", D. E., t. IV, Paris, Gallimard, 1994, pp. 631-636.

16 Sigo en esto el trabajo de Roth, X., op. cit.
} 
La invocación, ya en Alain, de una materia resistente al espíritu como paso obligado, anuncia un motivo que tendrá mucho porvenir, tanto en Canguilhem como en Foucault: la primacía de lo negativo, del error, de la enfermedad, de la locura, respecto a una verdad, un estado normal y una racionalidad que son siempre segundos, derivados.

Canguilhem se educó en esa filosofía del juicio, bajo el magisterio de Lagneau y sobre todo de Alain. Sin embargo, mediada la década de los años treinta, al mismo tiempo que empezaba a dudar de la fe pacifista inculcada por su maestro Alain, iniciaba un viraje, en cuyos detalles no podemos entrar ahora, que le llevó del juicio a la filosofía de la acción. Esta, con el elemento de incertidumbre, contingencia y error que le es inherente, aparecía entonces como una instancia previa al juicio y a su carácter universal y necesario. Inicialmente Canguilhem encaró el asunto de la acción en el terreno de la técnica y de su precedencia respecto a la ciencia. Frente a la vulgata positivista, recalcaba que la ciencia aparecía siempre en segundo lugar, como rectificación del error inmanente al tanteo técnico. La técnica, en cambio, se identificaba con la aventura y con la capacidad de configurar ficciones, se emparentaba así con la creación artística ${ }^{17}$. Al mismo tiempo recordaba que la técnica era una respuesta a necesidades y urgencias vitales.

Por esta vía, Canguilhem, que cursó en los primeros años 40 la carrera de Medicina al tiempo que se implicaba en la Resistencia francesa, transitó de la técnica a la vida como fundamento trascendental, unificador de la experiencia humana. En su tesis de medicina, Essai sur quelques problèmes concernant le normal et le pathologique, ya se había verificado este paso. La medicina es una técnica que prolonga, a través de la demanda del enfermo, el propio esfuerzo de la vida para resistir a la muerte, no sólo mediante la conservación y la adaptación al medio, sino sobre todo a través de la autocreación, de la instauración de normas que le permiten al viviente variar de entorno y conformarlo de un modo activo y selectivo ${ }^{18}$. La referencia decisiva, en este caso, la constituyó la filosofía biológica alemana (Driesch, Ricker, Von Uexküll, Von Weizsäcker, Goldstein), fuertemente inspirada en el neokantismo y en la filosofía de los valores ${ }^{19}$.

A continuación, examinaré de qué manera, tanto en Canguilhem como en Foucault, se afrontan las dos dimensiones cruciales del kantismo: el examen crítico de las condiciones de posibilidad de la experiencia y la apelación a una instancia fundadora, de orden trascendental. La relación respectiva con el polo crítico y con

\footnotetext{
17 Canguilhem, G.: "La création artistique selon Alain", Revue de Métaphysique et de Morale, 57 (1952), pp. 171-186, criticando los residuos platonizantes de las estéticas de Alain y Bergson.

18 Canguilhem, G.: Le normal et le pathologique, Paris, PUF, 1984, p. 153.

19 Schmigden, H.: "Georges Canguilhem et les 'discours allemands', en Fagot-Largeault, A., Debru, C. et Morange, M. (dir.), Han, H. (éd): Philosophie et medicine. En hommage á Georges Canguilhem, Paris, Vrin, 2008, pp. 49-62 y Roth, X., op. cit., pp. 30-31.
} 
el polo trascendental permitirá localizar mejor la diferente relación de ambos filósofos con el legado kantiano.

Tanto Canguilhem como Foucault emplazan el problema de la verdad en la vertiente crítica. El primero entiende que el discurso verdadero es patrimonio de la ciencia, pero al mismo tiempo considera que el valor de la verdad debe ser ponderado, en relación a la totalidad de los valores que constituyen la experiencia humana, es decir, en relación con la vida, fuente de todo valor. La actividad filosófica se identifica precisamente con esta tarea evaluadora ${ }^{20}$. Los límites y las condiciones de posibilidad del discurso científico se encuentran perfilados por el movimiento mismo de la vida, entendida como normatividad, esto es, como capacidad de creación de normas, de variaciones continuas que permiten a la individualidad viviente cambiar de medio, trascender el entorno dado.

La normación interna que caracteriza al discurso verdadero de la ciencia surge como una corrección de esta proliferación errática, engendradora de errores, que caracteriza al viviente. El conocimiento comienza con el error porque se arraiga en la vida. La técnica y la construcción de máquinas, como el arte, no constituyen sino una prolongación de esta creatividad vital21. La epistemología y la historia de las ciencias se subordinan, en Canguilhem, a su filosofía de la vida. Reconstruir la génesis de un concepto científico consiste en analizar las condiciones técnicas, institucionales y culturales a partir de las que se ha formado, en una relación que es a la vez de filiación y de ruptura. Se trata de recomponer, en una historia juzgada, evaluada, cómo un concepto científico se instaura dotándose de sus propias y autónomas normas de veridicción, rompiendo con ese magma de errores que lo anclan en la dinámica vital y que le impiden comprender esta misma dinámica. De este modo, el análisis de los límites funciona al mismo tiempo como una crítica de las ilusiones; así acontece con el mecanicismo o con el vitalismo metafísico, filosofías que quedan presas de barreras metafóricas (la asimilación del organismo a la máquina) o de sustancializaciones abusivas (apelación a un "impulso vital" que trasciende a los propios vivientes).

Foucault comparte con Canguilhem esta voluntad de mostrar los límites y las condiciones históricas de posibilidad de los discursos verdaderos o "juegos de verdad". Pero su interés no se dirige hacia las normas probatorias que distinguen al decir científico, sino hacia las regularidades (el "régimen de verdad") que permiten individualizar formaciones discursivas en el escenario del saber. Ese escenario,

\footnotetext{
20 Véase la intervención de Canguilhem en el debate radiofónico (junto a Foucault, Hyppolite y Ricoeur, entre otros) celebrado en 1965 y recogido en Foucault, M.: "Philosophie et verité", D.E., t. I, ed. cit., pp. 448-464. Véase también, Macherey, P.: "Canguilhem et la philosophie", en Ferté, L., Jacquard, A. et Vermeren, P. (dir), op. cit., pp. 27-42.

21 Canguilhem, G.: "Machine et organisme", en La connaissance de la vie, Paris, Vrin, 1980, pp. 101127.
} 
desde la década de los setenta, se convierte también en el de las tecnologías de poder.

Foucault reconoce, en La arqueología del saber, que una formación discursiva puede dotarse de la unificación conceptual y de las normas internas de veridicción que le permitan traspasar el umbral de epistemologización o de cientificidad, como sucede con las nociones estudiadas por Bachelard y Canguilhem. Por otro lado, en Vigilar y Castigar señala que una disciplina puede independizarse de la matriz político-discursiva en la que se formó (por ejemplo las ciencias naturales a partir de la práctica de la enquête), dotándose de sus propias normas internas de verdad 22 . Es decir, lejos de desembocar en formulaciones relativistas en el plano epistémico, la perspectiva arqueogenealógica de Foucault resulta concordante aunque distinta respecto a la historia epistemológica de Canguilhem.

Por otra parte, también en Foucault el análisis crítico de las condiciones de posibilidad tiene como consecuencia el desenmascaramiento de ilusiones dogmáticas. La tríada arqueológica compuesta por la Historia de la locura, El nacimiento de la clínica y Las palabras y las cosas, pone fuera de juego, respectivamente, la ilusión psiquiátrica que identifica a la enfermedad mental con un objeto natural; la ilusión médica que postula la eternidad de la clínica y la ilusión antropológica que identifica al hombre con un perenne objeto (y sujeto) del saber. Por otra parte, Vigilar y castigar y La voluntad de saber desmantelan, en el primer caso la ilusión jurídicopenal del delincuente y de la institución carcelaria como instancias transhistóricas, y en el segundo, la ilusión sexológica, avalada por la "hipótesis represiva", que postula la existencia del instinto sexual como invariante antropológica.

La convergencia y complementariedad, preservando las diferencias, entre arqueogenealogía e historia epistemológica en la dimensión crítica, no se repite, sin embargo, en la dimensión trascendental. Aparentemente Canguilhem parece rechazar el trascendentalismo kantiano, sustituyendo las estructuras a priori del conocimiento, universales y necesarias, por normas de racionalidad científica surgidas en un proceso puramente histórico, a través de un decurso gobernado por el error y su rectificación. Pero al identificar la raíz de la creación de errores con la normatividad vital, el filósofo de Castelnaudary parece topar con una suerte de trascendental objetivo o material23. En efecto; en su favorable reseña de Las palabras y las $\cos ^{2}{ }^{24}$, Canguilhem tomó conciencia del significado de su propia trayectoria, de su

\footnotetext{
22 Foucault, M.: Surveiller et punir. Naissance de la prison, Paris, Gallimard, 1975, pp. 227-228.

23 Roth, X., op. cit., pp. 135-150; Gayon, J.: "The concept of individuality in Canguilhem's philosophy of biology", Journal of the History of Biology, 31 (1998), pp. 305-325, p. 323 y Dagognet, F.: Georges Canguilhem, philosophe de la vie, Le Plessis-Robinson, Institut Synthélabo, 1997, p. 78, aunque no le seguimos cuando contrapone el "examen ontológico" de Canguilhem a los "estudios genealógicos" de Foucault.

${ }^{24}$ Canguilhem, G.: “¿Muerte del hombre o agotamiento del Cogito?”, en Burgelin, P. et Al.: Análisis de Michel Foucault, Buenos Aires, Tiempo Contemporáneo, 1970, pp. 122-147.
} 
tránsito, mediada la década de los años treinta, desde el idealismo kantiano que representaba Alain, hacia un vitalismo racionalista. Esta operación estaba perfectamente identificada en la obra de Foucault: Canguilhem había sustituido el sujetoCogito, la subjetividad trascendental kantiana por una de las estructuras empíricas nacidas en el paso de la episteme clásica a la moderna: la Vida. Esta actuaba en Canguilhem como una suerte de a priori objetivo, ocupando la función vacante de fundamento trascendental.

La estrategia teórica de Foucault parecía seguir, en este asunto, un derrotero completamente distinto al de su maestro. La enunciación más rotunda de su postura aparece en el debate que mantuvo en 1972 con el filósofo italiano Giulio Preti:

Comprendo su posición, pero es justo en este punto donde divergen nuestras posiciones. Usted me parece kantiano o husserliano. A lo largo de toda mi investigación yo me esfuerzo en cambio para evitar toda referencia a ese trascendental, que sería una condición de posibilidad para todo conocimiento. Cuando digo que me esfuerzo en evitarlo no afirmo que esté seguro de conseguirlo. Mi manera de proceder en ese momento, yo diría que es de tipo regresivo; intento asumir un distanciamiento cada vez mayor para definir las condiciones y las transformaciones históricas de nuestro conocimiento. Trato de historiar al máximo para dejarle lo menos posible a lo trascendental. Pero no puedo eliminar la posibilidad de encontrarme, un día, frente a un residuo no prescindible, que será lo trascendental. 25

Si Canguilhem, en último término, parece identificar la vida con el suelo trascendental descubierto por su filosofía (más adelante veremos hasta qué punto esto es problemático), la tarea de Foucault, en cambio, se encamina prima facie en una dirección inversa: sustituir lo trascendental por configuraciones praxeológicas (prácticas discursivas y no discursivas) históricamente mudables. Sin embargo, hay que recalcar que el fragmento citado no supone una exclusión por principio de lo trascendental. ¿Podría ser la vida ese "residuo" irreductible a la corrosión "eventualizadora" (en el sentido que da Foucault al verbo evénementialiser ${ }^{26}$ ) operada por el análisis arqueogenealógico?

\section{Un vitalismo que no quiere decir su nombre}

De entrada no. La vida, cuya exigencia en el plano arqueológico es rastreada en Las palabras y las $\operatorname{cosas}^{27}$, y cuyo comienzo en la escena genealógica ponen de

\footnotetext{
25 Foucault, M.: “Les problèmes de la culture. Un débat Foucault-Preti”, D. E., II, ed. cit., p. 373. La traducción es nuestra.

26 Foucault, M.: "La poussière et le nuage", $D E$, t. IV, ed. cit., pp. 24-25.

27 Foucault, M.: Les mots et les choses. Une archeologie des sciences humaines, París, Gallimard, 1966, pp. 275-292.
} 
relieve los análisis acerca de la biopolítica, no es una instancia trascendental. Se trata, sin más, de un "indicador epistemológico" 28 que acompaña al recorte de una nueva región en el saber moderno; la que se ocupa de los organismos vivientes como conjunto de funciones que resisten a la muerte. Por otro lado, la vida no es sino el polo objetivo, como fenómenos de "población", del biopoder característico de la gubernamentalidad liberal29. En suma, la vida no traspasa en Foucault la condición de un "objeto de época" que funciona como correlato de ciertas formaciones discursivas y de ciertas tecnologías de gobierno.

Este argumento, que asigna un papel relevante pero históricamente restringido, a la vida en el pensamiento de Foucault, ha sido revisado críticamente por algunos trabajos recientes que comparten el énfasis en las convergencias ontológicas constatables entre Canguilhem y Foucault. Ninguno de esos trabajos, no obstante, se atreve a afirmar, como defiendo en el curso de esta exposición, que la "ontología esquivada" por Foucault coincida con el vitalismo racionalista de Canguilhem.

El trabajo de Antonella Cutro (2004), por ejemplo, está movido por el empeño de encontrar, en el conjunto de la obra de Foucault, incluido su último periplo acerca de las tecnologías del yo, la "insospechada relación con una filosofía biológica" 30 . El argumento del libro consiste en detectar las metamorfosis del problema de la "vida" en todo el itinerario foucaultiano. En esta indagación, como era de esperar, Cutro se topa con la insoslayable convergencia que anuda, no sólo en el plano metodológico sino en el ontológico, la filosofía de Foucault, con el vitalismo de su maestro.

Cutro sugiere incluso que el objetivo de su investigación es detectar la "membrana" 31 que hace posibles los numerosos y fructíferos intercambios teóricos entre ambos filósofos. Sin embargo, en su trabajo sigue resaltando la alergia y la incomodidad de Foucault con todo lo que pueda evocar las seguridades de la ontología. Así, a la exploración realizada por esta profesora de la Universidad de Salerno, excelente pero insuficiente a nuestro modo de ver, pueden hacérsele dos achaques. En primer lugar no parece percatarse de la afinidad que existe entre la ontología histórica de Foucault y la "vida", tal como la entiende Canguilhem, esto es, como pura contingencia y "errancia", como una suerte de fundamento "infundado". En segundo lugar, y a diferencia de lo que se señaló en relación con la lectura realizada por Béatrice Han, Curto no se da cuenta de las tensiones internas, por no llamarlas inco-

\footnotetext{
28 Foucault, M.: "De la nature humaine: justice contre pouvoir", D.E., t. II, p. 474.

29 Foucault, M.: Securité, territoire, population. Cours au Collège de France. 1977-1978, Paris, Hautes Études, Gallimard, Seuil, 2004, pp. 359-365.

30 Cutro, A., op. cit., p. 12. No discutiremos aquí el trabajo de López, C., op. cit., pp. 255-260, que se limita a señalar las reticencias de Foucault ante el vitalismo y frente a toda tentativa de fundación ontológica.

31 Id., p. 18.
} 
herencias, que se derivan de una versión totalmente desontologizada del pensamiento de Foucault.

La perspectiva de Le Blanc (2002), uno de los mejores conocedores de la obra de Canguilhem ${ }^{32}$ y un cualificadísimo intérprete de la de Foucault ${ }^{33}$, sí llega a reconocer la presencia, eso sí, puntual e intermitente, del vitalismo en los escritos del filósofo de Poitiers. De entrada se constata la discrepancia entre maestro y discípulo. El primero afirma una creatividad normativa inherente al viviente humano, que actúa contrarrestando el poder regulador y extrínseco de la normalización social34. El segundo, sin embargo, considera que la subjetividad resulta completamente fabricada en el proceso de normalización; no es posible apelar a ninguna espontaneidad salvaje y exterior al ejercicio del poder 35 . No obstante, y aquí está lo "paradójico" de la tentativa foucaultiana, esta admite de forma ocasional la emergencia de "desvíos" (écarts) creativos que, rompiendo la lógica normalizadora, funcionarían como momentos de subjetivación y no de sujeción.

Le Blanc localiza estos eventos, testimonio de una vitalidad irreductible al ejercicio del poder, en tres registros puntuales del trayecto foucaultiano: el acto de pensar como desprendimiento de sí (entendido como comprensión de las normas de poder y de saber que nos gobiernan); la amistad como forma de vida (implicando la invención de nuevas relaciones éticas) y la revuelta (tomando como referencia la revuelta colectiva iraní o la experiencia individual de los "hombres infames"). En este último caso, de forma muy evidente, Foucault parece apelar a una creatividad vital que subtiende a la historia, una suerte de irrupción de la historia natural en la historia social ${ }^{36}$.

Pues bien, pese a reconocer este vitalismo subyacente, intermitente e inconfeso en el itinerario de Foucault, y aun admitiendo que esa noción de "desvío" creativo la toma de su maestro, Le Blanc sigue subrayando la incompatibilidad entre ambos proyectos filosóficos: la vitalidad es en Canguilhem una instancia permanente, mientras que en Foucault tiene el estatuto de una ocurrencia coyuntural. Pero entonces cabe preguntarse: la última etapa de Foucault, cuando se hace valer la potencia

32 Le Blanc, G.: Canguilhem et les normes, Paris, PUF, 1998 y Le Blanc, G., La vie humaine.., ed. cit, pp. 199-222.

33 Le Blanc, G.: El pensamiento Foucault, Buenos Aires, Amorrortu, 2008.

34 Canguilhem, G.: "Milieu et normes de l'homme au travail", Cahiers Internationaux de Sociologie, 23, 1947, 3, pp. 120-136, pp. 128-29, por ejemplo, entiende las reacciones obreras frente a la organización taylorista del trabajo, como "reacciones de salud".

35 Véase el rechazo explícito del "naturalismo" a partir de una pregunta realizada por Bernard HenriLévy, en Foucault, M.: "Non au sexe roi", D. E., t. III, pp. 264-65.

36 Le Blanc, La vie humaine.., op. cit., pp. 216-17. Rabinow, P.: "French Enlightenment: truth and life", Economy and Society, 27, 1998, 2-3, pp. 193-201, p. 193, señaló que en la obra de Canguilhem, el problema de la vida es una constante privilegiada, mientras que en Foucault, su presencia es más compleja e intermitente. 
autoformativa de la subjetividad a través de las prácticas de libertad, ¿constituye sólo un episodio puntual y restringido en la totalidad de la singladura foucaultiana?; ¿no surge entonces una contradicción difícilmente soluble desde la prioridad de la sujeción sobre la subjetivación?; ¿y no puede ser sorteada esa incoherencia reconociendo abiertamente la dependencia ontológica del pensamiento de Foucault respecto al vitalismo racionalista de Canguilhem?

Esta apelación, ocasional según Le Blanc, no abiertamente reconocida por Foucault, a una instancia ontológica que funcionaría como fuente de la resistencia y la creación, ha dado pie, ya desde los primeros textos arqueológicos de Foucault, a distintas valoraciones que resaltan las tensiones internas del pensamiento foucaultiano, derivadas de esta suerte de experiencia criptoontológica que gravita sobre su obra. Así, la invocación, en la Historia de la locura, de una "experiencia trágica" que operaría como locura ontológicamente originaria, suscitó las críticas de Derrida $^{37}$ y la detección de reminiscencias románticas en la opera prima del trayecto arqueológico38. Un destino similar han tenido las nociones de "afuera" y "pensamiento del afuera", un pensar transgresor de todo límite, que tiene su campo de acción en la literatura como experiencia del lenguaje en su ser en bruto, antes de toda función representativa. Esta experiencia remite a una especie de teología negativa, de pensamiento extático39. Algo parecido sucede con la noción de "plebe", inspirada en Glucksman y alusiva a un impulso originario de revuelta 40 , o con la referencia a "los cuerpos y los placeres" como punto de anclaje de la resistencia 41 . Estos conceptos han dado pie a sugerir un "naturalismo" "romántico" incorporado como una sombra insoslayable del periplo foucaultiano, pese a las tentativas del filósofo por escapar a ella.

37 Boyne, R.: Foucault and Derrida. The other side of reason, London, Unwin Hayman, 1990.

38 Dreyfus, R. y Rabinow, P.: Michel Foucault. Un parcours philosophique, Paris, Gallimard, 1984, p. 18; Hacking, I.: "The archaeology of Foucault" en Hoy, D.C. (ed.): Foucault. A critical reader, Oxford, Blackwell, 1986, p.29, y entre nosotros, Trías, E.: Filosofía y carnaval, Barcelona, Anagrama, 1979, p. 46 y Morey, M.: Lectura de Foucault, Madrid, Taurus, 1983, pp. 37-38.

39 Foucault, M.: El pensamiento del afuera, Valencia, Pre-Textos, 1988; Deleuze, op. cit., p. 116; Bernauer, J. W.: Michel Foucault's forcé of light. Towards an ethics for thought, New Jersey, Humanities Press, 1993, pp. 57-60; De Certeau, M.: "The black sun of language: Foucault", en Smart, B. (ed.): Michel Foucault. Critical Assessments, vol. II, New York and London, Routledge, 1994, pp. 246-258, pp. 255-56, y entre nosotros, Sahuquillo González, J.: Michel Foucault: una filosofía de la acción, Madrid, Centro de Estudios Constitucionales, 1989, pp. 140-161 .

40 Foucault, M.: "Pouvoir et stratégies", D.E., t. III, ed. cit., pp. 421-422 y Comay, R.: "Excavating the represive hypothesis: aporias of liberation in Foucault", en Smart, B. (ed.): Michel Foucault. Critical Assessments, vol. III, ed. cit., pp. 242-250, pp. 245-246.

41 Foucault, M.: La volonté de savoir. Histoire de la sexualité 1, Paris, Gallimard, 1976, p. 208; Foucault, M.: Herculine Barbin, llamada Alexina B., Madrid, Editorial Revolución, 1985, p. 11; Fraser, N.: "Foucault's body-language: a post-humanist political rethoric?", en Smart, B. (ed.): Michel Foucault. Critical Assessments, vol. III, ed. cit., pp. 3-13, pp. 6-7; Comay, op. cit., p. 246 y Butler, J.: El género en disputa, Barcelona, Paidós, 2010, pp. 197-198. 


\section{El vitalismo racionalista como vitalismo desfondado}

¿Qué es entonces lo que convierte al vitalismo de Canguilhem en el candidato idóneo para ocupar el lugar de esa "ontología esquivada" que atraviesa la obra de Michel Foucault? La "ontofobia" foucaultiana, su voluntad de radicalizar el nominalismo histórico dejando el menor lugar posible para lo trascendental, tiene que ver con un rechazo de todo esencialismo, sea este biológico o antropológico. En una ética, la de Foucault, que afronta la libertad como ejercicio de desposesión y experiencia de la fragilidad ("se dépendre de soi même") 42 , el esencialismo funciona evacuando la incertidumbre y el acontecimiento, cierra el horizonte de lo posible. Al mismo tiempo, el esencialismo, que en el planteamiento de Foucault parece asimilarse a toda ontología no histórica, es la justificación de un ejercicio del poder que tiende a la dominación, es decir, a bloquear toda práctica posible de la libertad.

Pues bien, por las características que reviste la vida en su argumentación, nada hay menos esencialista que el vitalismo canguilhemiano43. Su autor heredó de Alain y del kantismo la afirmación de la libertad como insumisión y resistencia frente a los hechos consumados. Este primado de la libertad se trasladó posteriormente, en paralelo a su proyección en la Medicina y en la Resistencia contra la ocupación, desde una filosofía del juicio y del espíritu hacia una filosofía de la vida.

Lo que distingue a los individuos vivientes según Canguilhem, es su relación polar con el entorno 44 . La individualidad biológica, desde las bacterias hasta los grandes mamíferos, se configura a partir de una constante preferencia y selección de los estímulos que proceden del entorno. Su inserción en el medio no es pasiva; consiste en un nexo de debate y evaluación permanente, que le lleva a configurar activamente ese medio ${ }^{45}$. Por otro lado, esta polaridad se despliega en un doble movimiento: de supervivencia por autoconservación, manteniendo constante el equilibrio del medio interno en relación con el externo, y de instauración de normas que permiten al viviente variar de entorno46.

Esta definición de la vida como normatividad vital, como capacidad de creación de nuevas normas, es central. Se expresa en primer lugar como "labilidad", plasticidad creadora del viviente, que se autoengendra a sí mismo a través de la proliferación de variaciones, del derroche de formas ${ }^{47}$, frente a toda apelación esencialis-

\footnotetext{
42 Bernauer, op. cit., p. 23 y Rachman, J.: Erotique de la vérité. Foucault, Lacan et la question de l'éthique, Paris, PUF, 1994, pp. 141-156. Bouchindhomme, C.: "Foucault, la moral y la crítica", en Balibar, E. et al.: Michel Foucault, filósofo, Barcelona, Gedisa, 1990, p. 315, identifica esta libertad con la "dimensión ontológica" que opera como base normativa de los análisis foucaultianos.

43 Sobre el "humanismo no esencialista" de Canguilhem, cfr. Macherey, P.: "Canguilhem et la philosophie", op. cit., p. 33.

44 Canguilhem, G.: Le normal et le pathologique, op. cit., pp. 78-79.

45 Canguilhem, G.: La connaissance de la vie, Paris, Vrin, 1980, pp. 146-47.

46 Canguilhem, G.: Le normal et le pathologique, op. cit., p. 87.

47 Id., p. 133, cfr. Le Blanc, L.: La vie humaine, op. cit., pp. 89-91.
} 
ta a una naturaleza o a un esquema de constancia que trata de mantenerse a toda costa. En el vitalismo de Canguilhem, la fuerza tiene prioridad sobre las formas que engendra. No cabe tampoco referirse a una "naturaleza humana"48. La vida biológica y social no consiste en el despliegue de unas "disposiciones naturales". Por el contrario, las normas, las disposiciones, son un resultado, en continua renovación y apertura, del modo en que el viviente singular se constituye creando normas en relación con un medio que tiende a variar. La vida, esto es, el proceso de individualización biológica, precede y crea las disposiciones. Así, el pez payaso macho puede cambiar de sexo cuando hay carencia de hembras, y el conejo puede transformarse en carnívoro ante la ausencia de hierba. No existe por tanto un modelo o estándar trascendente al que la vida se ajustaría.

En segundo lugar, como es bien conocido, Canguilhem cuestiona el dogma positivista que entiende lo patológico como mera variación cuantitativa respecto a lo normal ${ }^{49}$, pero también pone en tela de juicio la identificación de la enfermedad con la anormalidad, esto es, con la ausencia de normas. La diferencia cualitativa entre el individuo sano y el enfermo reside en la susceptibilidad para cambiar de entorno, instaurando nuevas normas vitales que reemplazan a las antiguas ${ }^{50}$. El enfermo se sostiene normativamente en su medio, siempre que este no se vea alterado. En la medida en que esta capacidad de rectificar las normas se encuentra reducida, hablamos de estado patológico; la salud sería una tolerancia ante las infidelidades del medio 51 .

En tercer lugar, Canguilhem descarta el concepto finalista de "desarrollo" a la hora de entender la temporalidad característica de la vida. Esta consiste en un proceso habitado por la "errancia", es decir, el riesgo y el error, el permanente ensayo creador de normas, un "devenir" abierto, una "aventura" que carece de telos alguno52. La vida se caracteriza pues por su contingencia histórica, se identifica con el surgimiento impredecible de eventos.

En cuarto lugar, la noción de vida incorpora en Canguilhem una ontología perspectivista $^{53}$. En efecto, la delimitación del estado sano o patológico no puede objetivarse en estándares normativos universales (por ejemplo los promedios de las constantes vitales), dado que exige analizar, en cada caso, la relación del viviente individual con su entorno. La vida es perspectiva, siempre situada y siempre singu-

\footnotetext{
48 Le Blanc, L.: La vie humaine, op. cit., p. 11 y Ebissienine, C. G.: La problématique de la santé et de la maladie dans la pensé biomédicale. Essai sur la normalité biologique chez Georges Canguilhem, Paris, L'Harmattan, 2010, p. 152.

49 Canguilhem, G.: Le normal et le pathologique, op. cit., pp. 11-67.

50 Id., pp. 132-133.

51 Id., p. 130.

52 Le Blanc, G.: La vie humaine, op. cit., p. 11.

53 Id., pp. 12-13 y Le Blanc, G.: "La vie selon ses points de vue", en Le Blanc, G.: Lectures de Canguilhem, op. cit., pp. 49-60.
} 
lar. Esto implica también su inmanencia; el vitalismo de Canguilhem excluye la referencia a un élan vital (al estilo de Bergson) o a un enigmático "principio vital" (al estilo de Stahl o más modernamente, de Teilhard de Chardin) que se expresaría en las formas individuales 54 .

La referencia a una vida entendida como sobreabundancia creativa de formas (y no como adaptación pasiva y utilitaria al medio), la invocación del perspectivismo, ¿no parecen revelar una coincidencia total entre los planteamientos de Nietzsche y de Canguilhem?

La filiación nietzscheana del vitalismo canguilhemiano es un asunto muy discutido por los comentaristas 55 . Canguilhem se consideraba a sí mismo como un "nietzscheano sin carnet" 56 , y es innegable la vecindad de su perspectivismo, de su noción de salud como creación artística de normas o de la primacía de la fuerza sobre la forma, con las posiciones del visionario de Sils-Maria. Pero quizás se haya exagerado el impacto de Nietzsche sobre Canguilhem, en detrimento de su dependencia respecto a la tradición francesa del análisis reflexivo o a la filosofía biológica y de los valores, de inspiración germánica.

Hay un punto capital de discrepancia y de incompatibilidad entre el vitalismo racionalista de Canguilhem y la filosofía de la voluntad de poder, una divergencia que se extiende asimismo, obviamente, al vitalismo deleuziano de las "máquinas deseantes". Pese a las vacilaciones que reflejan escritos relativamente tempranos, que los comentaristas emplazan en la etapa "ilustrada" de Nietzsche, como La gaya ciencia o Humano demasiado humano, Nietzsche parece ubicar la racionalidad científica, del lado de los ideales ascéticos y del nihilismo (o las "fuerzas reactivas", como las denomina Deleuze), esto es, de una vida resentida que se destruye a sí misma. Si la voluntad de verdad instaura una tregua o apaciguamiento más o menos violento de los instintos, el concepto científico opera como renuncia ascética, por abstracción (es decir, separando a la fuerza de lo que esta puede), respecto a las potencias afirmativas del cuerpo y de la sensibilidad.

\footnotetext{
54 Canguilhem, G.: "Aspects du vitalisme”, en La connaissance de la vie, op. cit., pp. 83-100; Han, H.J.: "Georges Canguilhem et le vitalisme français: remarques sur les 'aspects du vitaisme", en Fagot-Largeault, A., Debru, C. et Morange, M. (dir), op. cit., pp. 13-33 y Trnka, P.: "Subjectivity and values in medicine: the case of Canguilhem", Journal of Medicine and Philosophy, 28, 2003, 4, pp. 427-446, p. 443.

55 El análisis más completo lo presenta Stiegler, B.: "De Canguilhem á Nietzsche: la normativité du vivant", en Le Blanc, G. (ed.): Lectures de Canguilhem. Le normal et le pathologique, Fontenay -Saint Cloud, ENS Éditions, 2003, pp. 85-101. Véase también Cherlonneix, L.: "Après Nietzsche et Canguilhem", en Fagot-Largeault, A., Debru, C. et Morange, M. (dir.), Han, H. (éd), Op. cit., pp. $35-$ 47. No obstante, Roth, X., op. cit., pp. 121-123 cuestiona la excesiva importancia concedida al impacto de Nietzsche en Canguilhem.

56 Roth, X., op. cit., p. 121.
} 
La relación entre vida y conocimiento, entre vida y ciencia biológica, presenta en Canguilhem un aspecto completamente dispar 57 . Es un asunto que trató repetidas veces a lo largo de su vida, y que fue modulando gradualmente desde la segunda mitad de los años cuarenta (cuando vieron la luz los primeros trabajos contenidos en La connaissance de la vie) hasta el texto decisivo de 1966, "Le concept et la vie". Las discrepancias principales pueden resumirse en dos enunciados.

En primer lugar, el conocimiento no es la negación de la vida, sino la prolongación, mediante el distanciamiento respecto al saber práctico inmediato, de las estrategias vitales que permiten resolver las tensiones entre el viviente y su entorno:

No es cierto que el conocimiento destruya la vida, sino que deshace la experiencia de la vida con el fin de abstraer de ella, por el análisis de los fracasos, unas razones de prudencia (sabiduría, ciencia, etc) y unas leyes de éxitos eventuales, en vistas de ayudar al hombre a rehacer esto que la vida ha hecho sin él, en él, o fuera de él.58

En segundo lugar, en un momento posterior de su singladura intelectual, Canguilhem aproximó la genética y el aristotelismo: la racionalidad no sólo no constituye una negación de la vida, sino que se identifica con un Logos inscrito en la nervadura misma de los seres vivos, en la forma de un código que rige la replicación y transmisión del material génico ${ }^{59}$. Esta inmanencia de la racionalidad en la vida es lo que hace posible el conocimiento biológico; es decir, la vida no es una fuerza misteriosa e impenetrable, al estilo del vitalismo romántico; tampoco se identifica con la "duración", contrapuesta a la espacialidad del concepto científico, como en Bergson60. Se trata de un ámbito accesible a la comprensión racional, en tanto las formas de racionalidad no son heterogéneas respecto a las formas vivientes, sino que se alojan en las mismas bases moleculares de la dinámica vital. Esto le permite a Canguilhem concebir la historia de las ciencias sobre la imagen del propio movimiento vital, como producción y rectificación incesante de errores, como instauración de normas probatorias, de veridicción.

\footnotetext{
57 Sobre esta diferencia, Schwartz, Y.: "Une remontée en trois temps: Georges Canguihem, la vie, le travail", en AAVV.: Georges Canguilhem, philosophe.., op. cit., pp. 305-321, p. 311 y Debru, C.: Georges Canguilhem, science et non-science, Paris, Éditions Rue d'Ulm, 2004, pp. 86-87.

58 Canguilhem, G.: La connaissance de la vie, op. cit., p. 8. La traducción es nuestra.

59 Canguilhem, G.: "Le concept et la vie", en Études d'histoire et de philosophie des sciences, Paris, Vrin, 1983, pp. 335-364. Sobre la incompatibilidad de esta tesis con el vitalismo nietzscheano, Le Blanc, G.: La vie humaine.., op. cit., pp. 278-79.

60 Este no antagonismo entre vida y conocimiento distingue la posición de Canguilhem respecto a las de Nietzsche, Bergson y las filosofías de la existencia. Véase Worms, F.: "Le concept du vivant comme philosophie premiére: de Canguilhem á aujourd'hui”, en Fagot-Largeault, A., Debru, C. et Morange, M. (dir.), Han, H. (éd), op. cit., p. 140 y Dagognet, F.: Georges Canguilhem, op. cit., 182.
} 
Ahora bien, la "ontología esquivada" por Foucault, ¿concuerda más con el vitalismo racionalista de Canguilhem 61 o con el vitalismo nietzscheano? Deleuze ${ }^{62}$, sin entrar a examinar la primera alternativa, asimila el "vitalismo" de Foucault -vinculado a la "capacidad de resistir" propia de una "fuerza procedente del afuera", a la versión nietzscheana. Pero los estudios arqueogenealógicos no autorizan a derivar el diagnóstico nietzscheano que presenta a la ciencia como una fuerza negadora de la vida. Las ciencias cuyo estatuto epistemológico queda debilitado a partir de los análisis de Foucault, son disciplinas "inmaduras", como las llama Hacking63, cuyas reglas de formación no han atravesado ni el umbral de epistemologización ni mucho menos el de cientificidad ${ }^{64}$ : psicología, psiquiatría, criminología, pedagogía, ciencias de la sexualidad, psicoanálisis, etc. Curiosamente, coinciden en buena medida, al menos en algunas circunstancias, con las disciplinas cuestionadas por Canguilhem; este es sobre todo el caso de la psicología 65 . Ambos filósofos comparten la crítica de saberes que funcionan como pura legitimación del orden de cosas establecido, que predican, siguiendo una expresión cara a Canguilhem, la "adoración de los hechos" 66 .

Frente a esas disciplinas "inmaduras" se levantan aquellas cuyo desbloqueo epistemológico, según Foucault, les ha permitido independizarse de su matriz jurídico-política inicial, dotándose de sus propias normas autónomas de validez. Aquí se insertan, obviamente, ciencias empíricas como la Física, la Química y las Ciencias de la Vida. Aunque Foucault no se ocupa directamente de ellas, sí le interesan, especialmente por sus consecuencias sociales. Así por ejemplo, reseñando el libro Jacques Ruffié, De la biologie á la culture, resalta de qué modo "un saber científico riguroso" 67 como la genética y la biología molecular, ha contribuido a

${ }^{61}$ El rótulo de "vitalismo racionalista" para designer la filosofía de Canguilhem, fue puesto en circulación por Rabinow, P.: "Introduction: a vital rationalist", en Delaporte, F.(ed.): A vital rationalist, op. cit., pp. 11-22.

62 Deleuze, G.: Foucault, op. cit., pp. 119-122.

63 Hacking, I.: "Foucault's inmature science", Nous, 13, 1979, 1, pp. 39-51.

64 Foucault, M.: Archéologie du savoir, Paris, Gallimard, 1969, pp. 243-251.

65 Roudinesco, E.: "Situation d'un texte: qu'est-ce que la psychologie?", en AAVV.: Georges Canguilhem, philosophe.., op. cit., pp. 135-144, p. 142.

66 Esto se advierte ya en un texto de juventud (1927): "Le fait n'a point par lui-même de valeur. Et même, du moment qu'il existe comme fait, c'est qu'il porte avec lui ses conditions. Les conditions, qui les connait les change. Aussi le fait traduit-il non pas ce qu'on fait, mais ce qu'on ne fait pas" (Canguilhem, G.: "La mobilization des intellectuels- protestations d'étudiants", en Écrits philosophiques et politiques, 1926-1939. Oeuvres completes, t. I, ed. cit., p. 167). Sobre la crítica de Canguilhem a la psicología por predicar la sumisión a los hechos, cfr. Braunstein, J. F.: "Jeunesse de Georges Canguilhem", en Ferté, L., Jacquard, A. et Vermeren, P. (dir.), op. cit., pp. 145-170; sobre sus convergencias con el joven Foucault, Moreno Pestaña, J. L.: En devenant Foucault. Sociogénèse d'un grand philosophe, Paris, Éditions du Croquant, 2006, pp. 235-241.

${ }^{67}$ Foucault, M.: "Bio-histoire et bio-politique", D. E., t. III, ed. cit., p. 96. 
disolver la idea de "raza". Foucault coincide con la definición antiesencialista de la vida que propone Canguilhem, al recalcar que una especie o una población no se definen por un prototipo o naturaleza, sino por un "conjunto de variaciones" 68 .

La posición de Foucault, resulta pues completamente ajena a cualquier forma de requisitoria contra la ciencia; ni "anarquismo epistemológico" a lo Feyerabend, ni afinidad con la "antimedicina" a lo Ivan Illich ni hiperconstruccionismo social a lo Bruno Latour. En cierto modo la experiencia del lyssenkismo ${ }^{69}$ curó a Foucault de espanto respecto a toda forma de crítica ideológica radical dirigida hacia el conocimiento científico. Esto explica, por ejemplo, que el alegato de Sokal y Bricmon contra el relativismo anticientífico de los filósofos postmodernos, no tomara como blanco a Foucault, y sí a autores como Deleuze, Derrida, Baudrillard o Lyotard70. Los planteamientos de éste resultan más afines con un racionalismo como el de Canguilhem -un racionalismo compatible con el reconocimiento de formas múltiples de racionalidad- que con el diagnóstico de la ciencia ofrecido por Nietzsche.

No se trata con ello de limar todas las asperezas entre Foucault y Canguilhem, pero sí de subrayar en qué medida la indecisión ontológica del discípulo genera tensiones e inconsistencias que quedan mejor resueltas por el maestro. Por otro lado, la ontología canguilhemiana no sólo es compatible con el antiesencialismo de Foucault, afirmando la vida como creación contingente y sobreabundante de variaciones y formas, sin teleología ni prototipo, sino que la instancia trascendental que invoca tiene la condición de un fundamento "infundado", donde se disuelve toda fijeza óntica, incluida la del devenir mismo ${ }^{71}$. En este sentido, la tentativa de Canguilhem, como la de Foucault, atraviesan una experiencia de "desfondamiento" y apuntan por ello a "narrar el abismo" (Abgrund) $)^{72}$.

En esa experiencia que constituye la vida como normatividad y errancia, el sujeto filosófico siempre está cogido antes de toda reflexión. Es decir, no hay apertura a la verdad ni descubrimiento filosófico; la filosofía está siempre de antemano

68 Esto no significa que la reflexión foucaultiana sobre la biología coincida en todo con la de Canguilhem. Reseñando el libro de François Jacob, La logique du vivant, Foucault señala que la genética actual se sitúa a una escala, la del mecanismo replicativo del ADN, previa a toda individualidad biológica (Foucault, M.: "Croître et multiplier", D. E., t. II, p. 101). Canguilhem sin embargo considera que la racionalidad biológica apunta siempre, en último término, a la relación entre una individualidad y su medio.

69 Moreno Pestaña, J. L., op. cit., pp. 98-107 y 231-235.

70 Sokal, A. y Bricmont, J.: Imposturas intelectuales, Barcelona, Paidós, 1999.

${ }^{71}$ Barrios, M.: La voluntad de poder como amor, Madrid, Arena Libros, 2006, p. 155.

72 Barrios, M.: Narrar el abismo. Ensayos sobre Nietzsche, Hölderlin y la disolución del clasicismo, Valencia, Pre-Textos, 2001, pp. 15-35. Esto ya había sido avistado por Foucault: "las reflexiones sobre la vida, el trabajo y el lenguaje, en la medida en que valen como analíticas de la finitud, manifiestan el fin de la metafísica" (Foucault, M.: Les mots et les choses, op. cit., p. 328; Las palabras y las cosas, México, Siglo XXI, 1974, p. 309). 
inserta en la vida y, dada la polaridad de ésta, es desde el principio evaluación y no investigación. La propia verdad, patrimonio exclusivo del conocimiento científico según Canguilhem, debe ser valorada por la filosofía en relación al conjunto de valores que componen la experiencia vital. Que las modernas ontologías de lo impensado -en el caso que nos ocupa, ese impensado es la vida- sean desde su inicio e inexorablemente un modo de acción, fue algo ya señalado por Foucault en Las palabras y las $\operatorname{cosas}^{73}$. Por eso en Canguilhem no hay ontología sin acción, por eso su filosofía, antes que epistemología o historia de las ciencias, se sostiene en el primado de la razón práctica.

\section{Conclusión. Normatividad vital y biopoder}

A continuación y para concluir seguiremos, en ese registro práctico, ético-político, el problema de la relación entre las normas sociales y vitales tal como se expone en ambos pensadores. Los recientes trabajos de Maria Mühle (2008, 2010 y 2012) y Gonzalo Velasco (2013) 74 , han puesto de relieve el papel capital desempeñado por la obra de Canguilhem en la génesis del concepto foucaultiano de biopolítica. Han señalado asimismo la deuda foucaultiana hacia un concepto de vida no esencialista, lo que les ha permitido distinguir la biopolítica foucaultiana respecto a los planteamientos esencialistas de Agamben, Negri y Hardt y Esposito.

Sin embargo, estos comentaristas, desatendiendo, tanto la invocación de una reactividad vital no subsumible por el ejercicio del poder, como el énfasis en las potencias autoformativas del sujeto, motivos ambos presentes en Foucault, se han aferrado a la defensa de un análisis de la biopolítica completamente desontologizado. Velasco considera que la autonomía de lo vital respecto a lo social, sostenida por Canguilhem, constituye una "ingenuidad"75 desde el punto de vista foucaultiano. Este supone que el ejercicio del poder carece de "afuera", produciendo al completo $\mathrm{y}$ sin resquicios las distintas formas de subjetividad.

Fue sin duda la lectura de El nacimiento de la clínica, lo que inspiró a Canguilhem un cambio decisivo en el modo de articular la relación entre normas vitales y normas sociales, pasando de una antropología regida por la biología a una biología regida por la antropología 76 . Debe admitirse también que la distinción foucaultiana entre un biopoder disciplinario y un biopoder securitario, afina y sofistica considerablemente el estudio del vínculo entre lo vital y lo social propuesto por Canguilhem. Pero al mismo tiempo todo conduce a afirmar, no sólo como señalan

\footnotetext{
73 Foucault, M.: Les mots et les choses, op. cit., p. 339.

74 Vid. Supra, nota 7.

75 Velasco Arias, G., op. cit., p. 257.

76 Le Blanc, G.: La vie humaine.., op. cit., p. 16.
} 
Mühle y Velasco, que la noción de vida de Foucault procede de Canguilhem, sino que el vitalismo del segundo está presente, aunque no desarrollado, en el primero, permitiendo además resolver la aparente incoherencia que supone el sujeto autopoiético invocado al final del periplo foucaultiano.

A partir de las "Nouvelles réflexions concernant le normal et le pathologique (1963-1966) ${ }^{77}$, Canguilhem reformuló su comprensión de las relaciones entre cuerpo viviente y cuerpo social. Las normas sociales no prolongan la creatividad vital, como hasta entonces había defendido; instauran un nuevo orden vital (una nueva allure de la vida $)^{78}$. No se trata de afirmar una ruptura dualista entre vida y sociedad, pues esta se apoya en aquélla, pero tampoco se sostiene ya un simple continuismo biologicista. Precisamente por su condición no de esencia sino de acontecimiento o variación, la creatividad vital se dice de muchas maneras ${ }^{79}$. La sociedad instaura normas imitando el propio movimiento vital ${ }^{80}$, pero esas normas, a diferencia de las orgánicas, no derivan espontáneamente de la relación del viviente con su medio; son el resultado, producto de luchas históricas, de un trabajo de normalización extrínseco respecto a lo normalizado. La norma no es en ese caso una creación que la individualidad biológica produce para poder variar el entorno. Se trata de un estándar impuesto desde afuera sobre los procesos ${ }^{81}$.

El concepto foucaultiano de biopoder implica también un mecanismo de mímesis. El ejercicio del poder sobre la vida opera imitando la propia lógica de los procesos vitales. Pero estos, como se deduce de la noción canguilhemiana de "polaridad", siguen un doble despliegue. Por una parte los individuos biológicos tienden a la constancia, a la autoconservación, preservando el equilibrio entre el medio interno y el externo. Por otro lado, los organismos funcionan inventando desvíos y errores, produciendo nuevas normas que les permiten cambiar de medio y al mismo tiempo autogenerarse; las anomalías no son por tanto fracasos sino ensayos vitales 82 . Como ha señalado Mühle83, el biopoder disciplinario imita el primer polo de

\footnotetext{
77 Véase también Canguilhem, G.: "Le problème des régulations dans l'organisme et dans la societé", en Écrits sur la médecine, Paris, Éditions du Seuil, 1989, pp. 101-124.

78 Canguilhem, G.: Le normal et le pathologique, op. cit., p. 137. Sobre este concepto, cfr. Le Blanc, G.: La vie humaine.., op. cit., pp. 240, 266 y Debru, C.: "Georges Canguilhem et la normativité du pathologique: dimensions épistémologiques et étiques", en AAVV.: Georges Canguilhem, philosophe..., op. cit., pp. 111-113.

79 Le Blanc, G.: La vie humaine.., op. cit., p. 107.

80 "Les phénomenes d'organisation sociale sont comme une mimique de l'organisation vitale, au sens oú Aristote dir de l'art qu'il imite la nature" (Canguilhem, G.: Le normal et le pathologique, op. cit., p. 188).

81 Id., pp. 185-191.

82 Daled, P. F.: “L'envers de la raison, du 'principe de Broussais' á Foucault via Canguilhem”, en Daled, P. F. (coord..): L'envers de la raison. Alentour de Canguilhem, Paris, Vrin, 2008, pp. 7-16, p. 14.

83 Mühle, M.: "Histoire(s) de la vie de Canguilhem á Foucault", op. cit., p. 195.
} 
lo vital, la tendencia a proteger, a preservar los equilibrios, encauzándolos a partir de una norma inducida desde el exterior con objeto de engendrar individuos dóciles y útiles. Estas tecnologías disciplinarias representan muy bien la manera de entender el nexo entre lo vital y lo social expuesta por Canguilhem ${ }^{84}$ : las normas sociales ajustan desde fuera la marcha de los procesos biológicos, dando lugar a un entorno planificado.

Pero Foucault, como es sabido, reconoce también la presencia de una segunda serie de mecanismos. Se trata de los dispositivos de seguridad. Aquí el modelo no es ya el de la "peste", 85 es decir, el de un poder que quiere evitar el desequilibrio por la adaptación de lo diverso a un estándar normativo externo. Opera en cambio el modelo de la "viruela". ${ }^{86}$ La desviación, la variación, el desequilibrio, no son instancias por corregir como en las disciplinas, sino instrumentos para un gobierno flexible de las poblaciones. Es decir, el biopoder no sólo imita a la vida en su vertiente de autoconservación y adaptación, sino también en su dimensión creativa, como fuente de variación, error, desvío. La posibilidad de un poder que mimetice la propia espontaneidad creadora de la vida no había sido prevista por Canguilhem. ¿Significa esto que no existe, en efecto, un "afuera” del poder?; ¿cómo explicar entonces las sublevaciones y resistencias?; ¿cómo dar cuenta de la potencia autoconstituyente de los sujetos?

Como la propia Mühle ha señalado, esa potencia de variación y desvío que caracteriza a los individuos vivientes, es imitada en efecto por los dispositivos de seguridad, pero sólo hasta un límite. En la medida en que las variaciones gestadas por la acción individual puedan ser alineadas con las metas del ejercicio del poder, aquellas se verán favorecidas e implementadas. ${ }^{87}$ Se trata sin duda de esos mecanismos de "traslación" o "interface" examinados en los Studies on governmentality, por los analistas anglosajones de la gubernamentalidad neoliberal.88 Se produce entonces una coordinación entre las acciones autónomas y autorreguladas de los

\footnotetext{
84 Sobre la anticipación del tema foucaultiano de la normalización disciplinaria en Canguilhem, cfr. Dagognet, F., op. cit., p. 51.

85 Foucault, M.: Les anormaux. Cours au Collège de France. 1974-1975, Paris, Hautes Études, Gallimard, Seuil, 1999, pp. 41-48, donde comenta además la noción canguilhemiana de "normalización".

86 Foucault, M.: Securité, territoire, population, op. cit., pp. 59-68.

87 Mühle, M.: "Sobre la vitalidad del poder: una genealogía de la biopolítica a partir de Foucault y Canguilhem", op. cit., pp. 427-428.

88 Sobre los mecanismos de translation, Rose, N.: Powers of freedom. Reframing political thought, Cambridge, Cambridge U.P., 1999, pp. 47-51. Este autor, tal vez el más representativo de los Studies on governmentality, considera que Foucault, a diferencia de Canguilhem, entiende la vida y el vitalismo como puros objetos de época, sin franquear nunca el paso de lo histórico a lo trascendental a la hora de afrontarlos (Rose, N.: "Life, reason and history: reading Georges Canguilhem today", Economy and Society, 27, 1998, 2-3, pp. 154-170, p. 164.
} 
individuos, y los fines de las acciones de gobierno. Este tipo de "acción a distancia" 89 permite entrelazar las tecnologías heteroformativas (de poder-saber) y las tecnologías autoformativas (de libertad), la política y la ética.

Pero la variación puede también funcionar como un enclave de contestación, romper su alineamiento con las tecnologías de gobierno y abrir un espacio diferente. Este nexo ambivalente, agónico, que Foucault expone como relación entre poder y libertad, queda mejor articulado, pues se impide así la recaída en el añejo dualismo entre espíritu libre y naturaleza determinada, en las claves del vitalismo canguilhemiano: la potencia creativa que despliegan los individuos biológicos en un medio socialmente normado, no se subordina sin más a las exigencias de autoconservación y equilibrio que las normas sociales imitan. Es el modo como los vivientes se engendran a sí mismos y gestan nuevos equilibrios desafiando los ya establecidos, convirtiéndose en agentes de cambio social.

Se ha tratado así, de reinscribir los problemas del análisis arqueogenealógico en la agenda del vitalismo y de la filosofía de la biología, con la intención de rebasar ciertas dicotomías (entre naturalismo y culturalismo, entre esencialismo y construccionismo, entre determinismo y libertad) que siguen atenazando hoy a las ciencias humanas y a los movimientos sociales a ellas vinculados. La incapacidad para encontrar en Canguilhem el trasfondo ontológico de los análisis de Foucault sólo puede deberse a cierto prejuicio teleológico que convierte necesariamente al maestro en un bosquejo imperfecto de lo que será el discípulo. El nuevo perfil de Canguilhem descubierto por la reciente literatura crítica obliga a rectificar este lugar común.

Francisco Vázquez García

Universidad de Cádiz

francisco.vazquez@uca.es

89 Vázquez García, F.: Tras la autoestima. Variaciones sobre el yo expresivo en la modernidad tardia, San Sebastián, Gakoa, 2005, p. 184. 